\title{
Renal Cancer Without Primary Cancer in the Kidney: Extra-Renal TFE3 Translocation Associated Renal Cell Carcinoma
}

\author{
Benjamin Petrinec ${ }^{\mathrm{a}, *}$, Bryan Morales Vargas ${ }^{\mathrm{b}}$, Lara R. Harik ${ }^{\mathrm{b}}$ and Viraj A. Master ${ }^{\mathrm{a}, \mathrm{c}}$ \\ ${ }^{a}$ Department of Urology, Emory University School of Medicine, Atlanta, GA, USA \\ ${ }^{\mathrm{b}}$ Department of Pathology, Emory University School of Medicine, Atlanta, GA, USA \\ ${ }^{\mathrm{c}}$ Winship Cancer Institute, Emory University, Atlanta, GA, USA
}

Received 13 October 2020

Accepted 18 February 2021

Pre-press 11 March 2021

Published 16 June 2021

\begin{abstract}
We report a case of an isolated para-aortic retroperitoneal renal cell carcinoma (RCC) in the absence of a primary cancer in the kidney. Single case reports in the literature have described extra-renal RCC in different locations with no evidence of primary renal tumor. We present the initial presentation, diagnostic imaging, surgical treatment, and pathologic evaluation. Immunohistochemistry demonstrated positivity for TFE3 and TFEB, both of which are Microphthalmia associated transcription factors (MiT) associated with translocation RCCs. We hypothesize these few cases of extra-renal RCC represent rare forms of translocation RCC.
\end{abstract}

Keywords: Renal cell carcinoma (RCC), extra-renal RCC, Microphthalmia associated transcription factors (MiTCase)

\section{CASE}

A 50-year-old Caucasian female presented to a local E.D. for abdominal pain. The patient denied hematuria, weight loss, night sweats or chills at that time. On physical exam, the patient exhibited flank tenderness, but no palpable mass. CT scan demonstrated a retroperitoneal mass. CT guided biopsy showed renal cell carcinoma with clear cell features. Our review of her outside scans revealed an $8 \mathrm{~cm}$ left retroperitoneal mass, with no evidence of lesions in the right or left kidney. The patient's past medical history was positive for obesity, with BMI of 41. ECOG score was 0 . The patient did not endorse any family history of kidney malignancy or any other type of cancer.

\footnotetext{
${ }^{*}$ Correspondence to: Ben Petrinec, MD, Urology Clinic, Building B, 1365 Clifton Road, Atlanta. GA 30322, USA. E-mail: bpetrin@emory.edu.
}

Following her initial visit, a repeat CT abdomen and pelvis (Fig. 1 and Fig. 2) with and without IV contrast was completed. The radiology report described a 'completely' exophytic left pararenal mass with involvement of renal vasculature. No clear involvement of the left kidney was described. The scan demonstrated left periaortic lymph node involvement and a normal right kidney. No evidence of distant metastasis was found. CT of the chest without contrast demonstrated small benign pulmonary nodules.

Three months later, our patient underwent an uncomplicated retroperitoneal mass resection with left nephrectomy. Tumor was noted to be invading the renal vein at the hilum as a renal vein tumor thrombus. Pathology review showed no carcinoma in the renal parenchyma or collecting system. None of the 12 lymph nodes dissected were positive for malignancy and all margins were reported as negative. Currently, the patient demonstrated no evidence of disease 6 


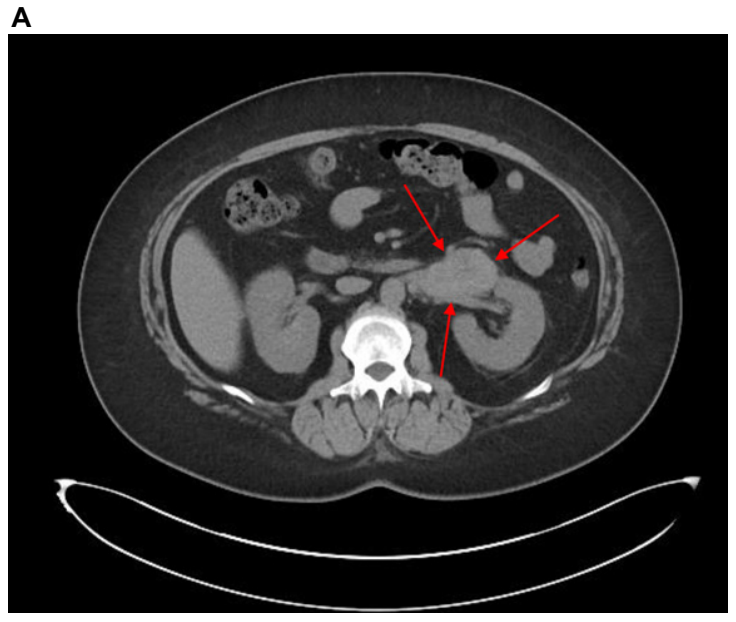

B

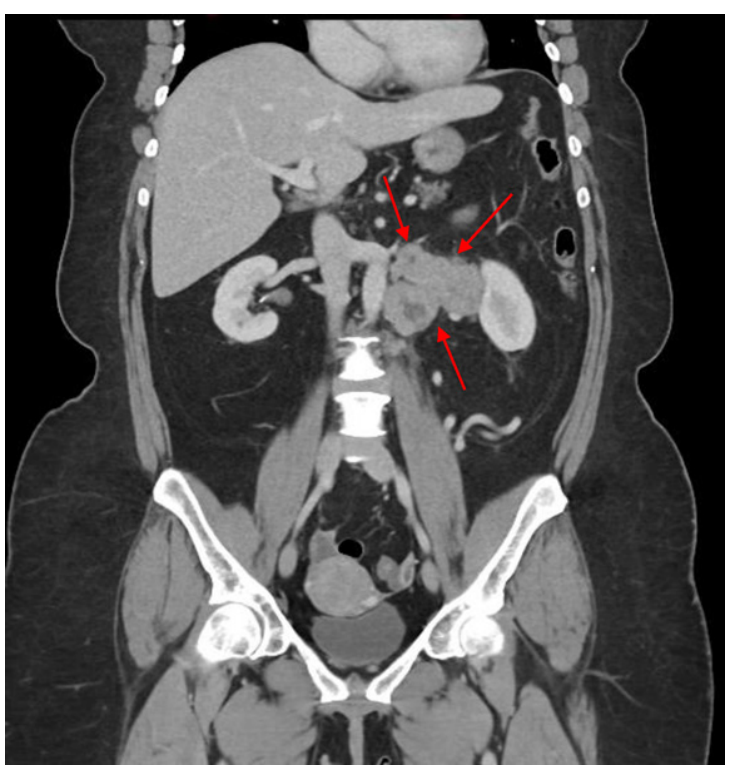

Fig. 1A. Pre-operative CT scan of left retroperitoneal mass, axial view. B Pre-operative CT scan of left retroperitoneal mass, coronal view. CT scan demonstrates left exophytic pararenal mass with involvement of renal vasculature. As discussed in the text, no clear involvement of the left kidney was evident. Left periaortic lymph node involvement and a normal right kidney can be appreciated.

months after surgery on cross sectional imaging of the chest, abdomen and pelvis.

\section{GROSS DESCRIPTION}

The left nephrectomy and retroperitoneal tumor resection, weighed $944 \mathrm{gm}$, on gross pathologic examination. Sectioning revealed an irregular multinodular dark red/black mass with intersecting fibrous bands, located outside the kidney, within the renal

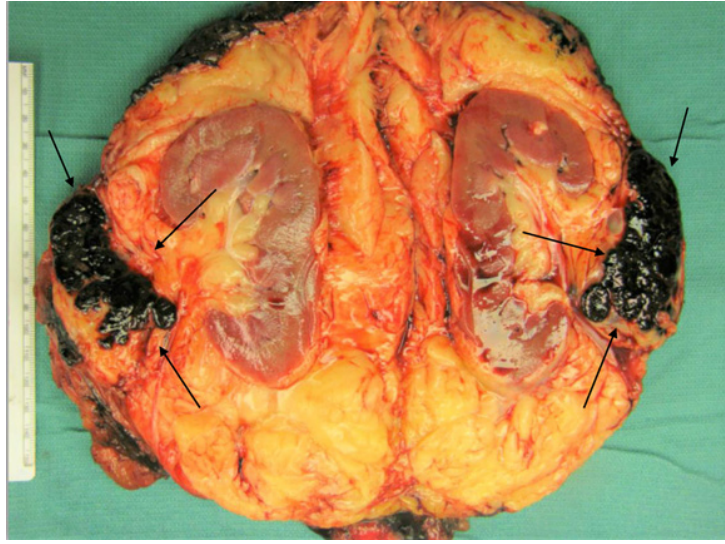

Fig. 2. Gross photograph of the renal tumor (black arrows) demonstrating its location within the renal hilar soft tissue, outside the renal parenchyma. Examination of the specimen demonstrated no involvement the kidney, which was otherwise unremarkable. Multiple sections to demonstrate the relationship of tumor to renal parenchyma were submitted and confirmed the carcinoma did not involve the renal parenchyma.

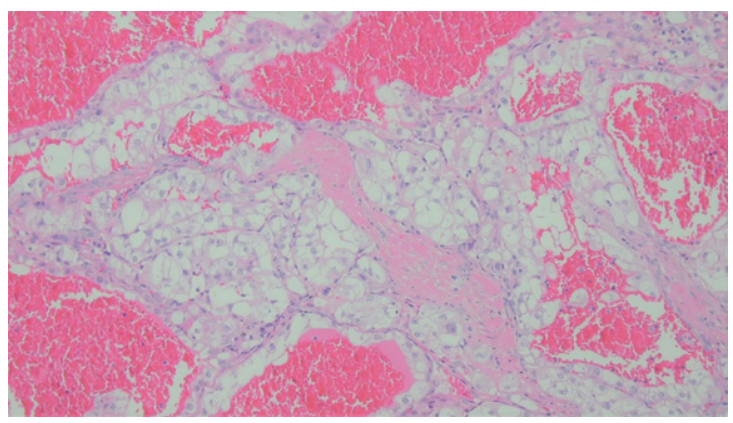

Fig. 3. Hematoxylin and Eosin Stain showed a tumor composed of clear cells arranged in nests, surrounded by delicate vasculature, and cysts engorged with blood.

hilum adipose tissue (Fig. 3). The mass measured $6.2 \times 5.5 \times 4.4 \mathrm{~cm}$, involved the renal sinus fat but did not involve the pelvicalyceal system or ureter. The mass invaded into the renal vein as a tumor thrombus. The mass did not arise from or involve the kidney, which was otherwise unremarkable. Multiple sections to demonstrate the relationship of tumor to renal parenchyma were submitted and confirmed the carcinoma did not involve the renal parenchyma.

\section{MICROSCOPIC DESCRIPTION}

Microscopically the tumor appeared encapsulated and was composed of clear cells arranged in trabeculae, nests and microcysts, separated by delicate vasculature, in a hemorrhagic background (Fig. 4). 


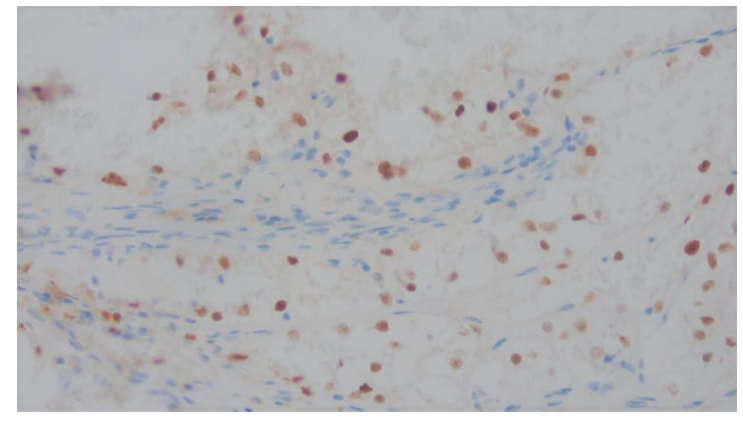

Fig. 4. The tumor was positive for PAX-8, a nuclear stain confirming renal origin.

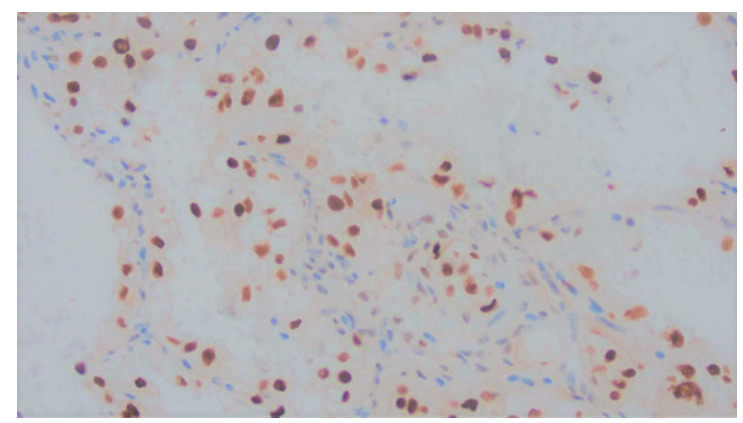

Fig. 5. Immunohistochemical stain for TFE3 showed nuclear positivity, in keeping with an equivocal result necessitating the exclusion of TFE3 associated renal cell carcinoma.

Neoplastic cells, had well-defined cytoplasmic membranes, with abundant clear cytoplasm, vesicular chromatin and prominent nucleoli, in keeping with WHO/ISUP nuclear grade 3. Histologically, the differential diagnosis was between clear cell renal cell carcinoma and translocation associated renal cell carcinoma.

The carcinoma was positive for cytokeratin AE1/ AE3, confirming an epithelial origin for this carcinoma, and PAX-8 nuclear positivity in keeping with renal origin (Fig. 5). Diffuse nuclear positivity for TFE3 (Fig. 6), an equivocal result, necessitated the exclusion of Xp11 translocation-associated renal cell carcinoma.

Thus, next-generation sequencing (NGS) using the Archer FusionPlex was performed on the Ilumina MiSeq instrument after RNA preparation. The Archer Sarcoma Fusion Panel 26, which assesses the fusion status of multiple genes involved in various sarcomas, detected a TFE3-ASPSCR 1 fusion, confirming the diagnosis of Xp11 translocation-associated renal cell carcinomas.

\section{DISCUSSION}

Few cases of primary extra-renal RCC have been reported. Several case reports have emerged that describe renal-type clear cell carcinoma (RTCCC) of the prostate. In these reports, patients present with hematuria or lower urinary tract like symptoms. Upon workup, these patients lack primary mass in either kidney, guiding authors towards the diagnosis of RTCCC rather than metastatic disease. Morphologically and histologically, these tumors are described to look like renal clear cell carcinoma [1-8]. Cases of primary extra-renal RCC arising in peri-nephric adipose tissue, the adrenal glands and adjacent to the inferior vena cava have been described [9-12]. Tumor characteristics and location from these cases are summarized in Table 1. Terada et al. (2011) reported a case in which a patient underwent nephrectomy for a small $1 \times 1 \times 1 \mathrm{~cm}$ lower pole renal mass which was located in perirenal adipose tissue [9]. The authors described no connection to the kidney or collecting system in any way. Authors describe the gross appearance of the tumor as hemorrhagic and solid. Histology and staining demonstrated clear cells positive for CD10,CD15, cytokeratin (CK) CAM5.2, CK AE1/3, CK18, a-methyl CoA racemase (AMACR), and Ki-67 (labeling index $=20 \%$ ) but negative for CK8, HMB45, inhibin, calretinin CD68, p53, TTF1 , and CDX2. The authors do not mention TFE3 [9]. Similarly, Hasan et al. (2015) report a case of clear cell RCC resected from an adrenal gland with no primary renal tumor [11]. Gross examination of this specimen was similar, with necrotic, hemorrhagic, fluid filled and cystic portions. The authors described a mass attached to the adrenal gland, separate from kidney, ureter, or renal arterial supply. On histological examination, tumor cells were described as polygonal in shape, and having clear or pale eosinophilic cytoplasm. Cells exhibited signs of high mitotic activity. Immunohistochemistry (IHC) of the tumor cells demonstrated positivity for CD 10 and CK (though weakly). Cells were negative for chromogranin, epithelial membrane antigen (EMA) and inhibin. A case described by Li et al. (2019) detailed the care of a patient in which RCC was located in the right lateral side of the inferior vena cava in the absence of lesions concerning for malignancy in either kidney [12]. Examination of the specimen revealed an $8 \mathrm{~cm} \times 7 \mathrm{~cm} \times 5 \mathrm{~cm}$ solid tissue with capsule on portions of its surface. On microscopy, the tumor tissue showed papillary arrangement and visible nucleoli. Results of IHC showed that tumor tissue 
Table 1

Summary of Tumor Characteristics from Extra-Renal RCC cases

\begin{tabular}{|c|c|c|c|c|}
\hline Author & Year & $\begin{array}{l}\text { Location } \\
\text { of Tumor }\end{array}$ & $\begin{array}{c}\text { TFE3/TFEB } \\
\text { Positivity }\end{array}$ & $\begin{array}{c}\text { Pathologic } \\
\text { Subtype }\end{array}$ \\
\hline Singh et al. & 2003 & Prostate & N/A & Renal-type Clear Cell Carcinoma \\
\hline $\begin{array}{l}\text { Pal and } \\
\text { Chowdhury }\end{array}$ & 2007 & Prostate & N/A & Renal-type Clear Cell Carcinoma \\
\hline Permi et al. & 2011 & Prostate & N/A & Renal-type Clear Cell Carcinoma \\
\hline Terada et al. & 2011 & $\begin{array}{c}\text { Peri-renal } \\
\text { adipose tissue }\end{array}$ & N/A & Clear Cell RCC \\
\hline Hasan et al. & 2015 & Adrenal gland & N/A & Clear Cell RCC \\
\hline Patne et al. & 2015 & Prostate & N/A & Renal-type Clear Cell Carcinoma \\
\hline Wang and Xue & 2015 & Prostate & N/A & Renal-type Clear Cell Carcinoma \\
\hline Sato et al. & 2016 & Prostate & N/A & Renal-type Clear Cell Carcinoma \\
\hline Liao et al. & 2018 & Prostate & N/A & Renal-type Clear Cell Carcinoma \\
\hline Han and Lim & 2020 & Prostate & N/A & Renal-type Clear Cell Carcinoma \\
\hline Li et al. & 2019 & $\begin{array}{l}\text { Adjacent to the } \\
\text { Inferior Vena Cava } \\
\text { (absence of lesion } \\
\text { in either kidney) }\end{array}$ & $\begin{array}{c}\text { TFE3 and } \\
\text { TFEB Positive }\end{array}$ & Type II Papillary RCC \\
\hline Petrinec et al. & 2021 & Retroperitoneum & TFE3 positive & Translocation RCC \\
\hline
\end{tabular}

expressed CD10, CAIX, ECAD, CK7, P504S, TFE3, TFEB, SDHB, CK20, Ki 67 (about 3\%), PAX-8 and PAX-2. This tumor was negative for Vim, CD117, Ksp-Cadherin. Pathologic diagnosis was type 2 papillary RCC. Of particular note, this tumor was positive for TFE3 and TFEB, but no further studies to rule out translocation associated renal cell carcinoma were reported [12]. Nunes et al. described a case of a patient undergoing adrenalectomy for an adrenal nodule. Pathologic diagnosis of the nodule was papillary RCC. IHC was positive for RCC Ma, CK7, CD10, and racemase and negative for CK20 and CD117. Both kidneys appeared normal on MRI imaging, and whole-body positron emission tomography was negative. A year later, the contralateral adrenal demonstrated a lesion that was diagnosed as papillary RCC [10].

Differential diagnosis during the course of workup for these extrarenal RCC masses can be complicated. Previous theories about the origin of extrarenal $\mathrm{RCC}$ with no renal primary tumor have ranged from remnant mesonephric duct to supernumerary kidney with concomitant RCC formation. Fetal renal development involves regression of the pronephros and mesonephros while the metanephros become the metanephric blastema, and eventually, the kidneys. It has been previously suggested that persistence of metanephric structures beyond gestation may provide renal tissue outside the kidney that is particularly susceptible to development of malignancy. The theory that extra-renal clear cell tumors arise from embryologic renal remnants within or adjacent to tissues such may arise from embryologic renal remnant in or adjacent to the prostatic, retroperitoneal, or adrenal tissue due to abnormalities of the urinary tract embryogenesis is most accepted $[9,10]$. Previous literature has described metastatic RCC to unpredictable locations such as the gallbladder or prostate [13]. Metastatic RCC with regression of primary cancer was suggested by some authors, but lack of primary renal tumor seems to rule this out in our patient and in previous extra-renal RCC case reports. Lastly, previous authors have suggested that the extra-renal RCC represents a supernumerary kidney which was transformed toneoplastic cells $[9,10,12]$.

Microphthalmia associated transcription factors (MiT) are a family of transcription factors that regulate melanocyte and osteoclast differentiation. These transcription factors are also associated with gene fusions identified in subtypes of RCC. MiTs includes TFE3, TFEB, TFEC, and MITF. One subset of these chromosomal translocations involve the Xp11.2 breakpoint. The most common Xp11 translocations affect TFE3 and have been reported as those which fuses the PRCC and TFE3 genes $(\mathrm{t}(\mathrm{X} ; 1)$ (p11.2;q21)), the $\mathrm{t}(\mathrm{X} ; 17)(\mathrm{p} 11.2 ; \mathrm{q} 25)$ which fuses the $A S P L$ and TFE 3 genes, and the fusion of $S F P Q$ and TFE3 (t(X;1) (p11.2;p34)) [14, 15]. Another subset of these translocations involving $\mathrm{t}(6 ; 11)$ represent the only described TFEB translocation, although recently there are reports of potential TFEB amplification [16].

The overexpression of TFE3/TFEB lead to transcription of lysosomal genes involved in several lysosomal processes, such as biogenesis and autophogagy, promoting formation of RCC $[17,18]$. Although translocation-associated renal cell carcinoma represent 
a higher percentage of renal tumors occurring in children and young adults, they have been reported in all age groups and the absolute number of this RCC subtype is higher in adults $[19,20]$. Previous literature has described indolent behavior, though more recent reports detail aggressive tumor behavior in adult populations [21]. Behavior is variable and may be related to the translocation. Some literature has suggested that these mutations may represent a higher percentage of RCC cases among adult and pediatric populations than originally expected. Studies have estimated that $3-4 \%$ of adult renal cell carcinomas are related to translocation abnormalities, while up to $40 \%$ of pediatric tumors may be related to these mutations $[22,23]$. Though male or female sex has not been thoroughly studied, one series of cases suggested that these translocations may occur predominantly in females (22 of 28 cases) [24]. Exposure to cytotoxic chemotherapy is thought to be a risk factor [20]. Gross appearance of these tumors has been relatively non-specific, with descriptions of tan or yellow masses, with hemorrhagic and necrotic areas. Histologic features are variable. Tumor cells have been described as clear, pale or granular, with large amounts of cytoplasm and eosinophilic granules. Cells are described as having alveolar, papillary or nested patterns with occasional psammoma bodies. Clear cell borders, prominent nucleoli, and mitotic figures have also been described. Previous cases have demonstrated that these translocation RCCs tend to express lower levels of epithelial immunohistochemical markers including cytokeratin and epithelial membrane antigen (EMA) in comparison to other RCC subtypes. Translocation RCCs frequently express melanocytic markers (Melan A, HMB45) and cathepsin K. Classical cytogenetics, $\mathrm{PCR}$, and Fluorescence in situ hybridization (FISH) can demonstrate translocations involving TFE3 or TFEB $[16,17]$. Resection, mTOR and tyrosine kinase inhibitors may be beneficial [20]. A study of 47 patients with translocation RCC by Wagner et al reported that selective MET inhibitors appear safe, but have modest antitumoral activity in this population [25]. Malouf et al. reported outcomes of vascular endothelial growth factor (VEGF) targeted therapy and mTOR inhibitory treatment for patients with translocation RCC. Among their 23 patients, treatment with sunitinib and/or mTOR inhibitors demonstrated progression free survival similar to rates reported for clear cell RCC patients [26]. Similarly, Choueiri et al. and Al-Daghmin et al. reported similar results for their respective cohorts of patients treated with VEGF targeted agents such as sunitinib [27, 28]. Another study reported that immune checkpoint inhibitors (ICIs) such as nivolumab may have a role in treatment for these patients. Among the translocation RCC patients the authors retrospectively analyzed, ICIs demonstrated similar rates of treatment efficacy to that of clear cell RCC patients. Additionally, the authors report that mutations of bromodomain member genes (PBRMI and BRD8) may be associated with increased benefit from ICI treatment [29].

\section{CONCLUSION}

Few cases of extra-renal RCC without renal primary tumor have been reported. The renal cell carcinoma subtypes were variable with clear cell RCC, papillary RCC and our case of TFE3 translocation associated renal cell carcinoma. $\mathrm{Li}$ et al. also reported on positivity or negativity of TFE3 or TFEB, however, additional studies were not reported [12]. Further studies to elucidate these rare tumors, including RCC subtype and pathogenesis are needed.

\section{INFORMED CONSENT}

Informed consent was obtained from the patient.

\section{CONFLICTS OF INTEREST}

The authors have no conflicts of interest to report.

\section{REFERENCES}

[1] Han SI, Lim S-C. Rare Case of Renal-type Clear Cell Carcinoma of the Prostate and Review of the Literature. In Vivo. 2020;34(5):2751-6. doi:10.21873/invivo. 12098

[2] Singh H, Flores-Sandoval N, Abrams J. Renal-type clear cell carcinoma occurring in the prostate. Am J Surg Pathol. 2003;27(3):407-10. doi:10.1097/00000478200303000-00016

[3] Wang Q, Xue Y. Renal-type clear cell carcinoma of the prostate: A case report. Oncol Lett. 2015;9(5):2149-52. doi:10.3892/ol.2015.3062

[4] Permi HS, Laxminarayana KPH, Yeshvanth SK, Shetty JK. Renal Type Clear Cell Carcinoma of the Prostate: A Diagnostic Dilemma. J Lab Physicians. 2011;3(2):132-3. doi:10.4103/0974-2727.86853

[5] Patne SCU, Johri N, Katiyar R, Trivedi S, Dwivedi US. Renal-type clear cell carcinoma of the prostate: a diagnostic challenge. Diagn Pathol. 2015;10(1):193. doi:10.1186/s13000-015-0432-8

[6] Sato Y, Kataoka M, Hata J, Akaihata H, Ogawa S, Kojima Y. Renal-type Clear Cell Carcinoma Occurring in the Prostate With Zinner Syndrome. Urol Case Rep. 2016;5:912. doi:10.1016/j.eucr.2015.11.008 
[7] Liao G, Zhang X, Li Z, Lan S, Huang M, Huang W. Renal-type clear cell carcinoma of prostate: A case report and review of literature. Indian J Pathol Microbiol. 2018;61(3):431-3. doi:10.4103/IJPM.IJPM_396_17

[8] Pal D, Chowdhury M. Renal type clear cell carcinoma of prostate. Indian J Surg. 2007;69(2):81-4.

[9] Terada T. Extra-renal clear cell renal cell carcinoma probably arising from mesodermal embryonic remnants. Pathol Int. 2012;62(4):291-3. doi:10.1111/j.14401827.2011.02780.x

[10] Nunes G, Pinto-Marques P, Sequeira P, Mendonça E. Primary Extrarenal Renal Cell Carcinoma: A Unique Diagnosis Performed through Endoscopic Ultrasound. GE Port J Gastroenterol. 2019;26(5):378-80. doi:10.1159/000496279

[11] Hasan R, Kumar S, Monappa V, Ayachit A. Primary extra-renal clear cell renal cell carcinoma masquerading as an adrenal mass: A diagnostic challenge. Urol Ann. 2015;7(4):513-5. doi:10.4103/0974-7796.162219

[12] Li Y, Qiu X, Li W, et al. Primary Extrarenal Type 2 Papillary Renal Cell Carcinoma: A Case Report. Urology. 2019;123:e1-e3. doi:10.1016/j.urology.2018.10.039

[13] King DH, Centeno AS, Saldivar VA, Sarosdy MF. Renal cell carcinoma metastatic to the gallbladder or prostate: two case reports. Urology. 1995;46(5):722-5. doi:10.1016/S00904295(99)80310-0

[14] Caliò A, Segala D, Munari E, Brunelli M, Martignoni G. MiT Family Translocation Renal Cell Carcinoma: from the Early Descriptions to the Current Knowledge. Cancers. 2019;11(8). doi:10.3390/cancers 11081110

[15] Wang X-T, Xia Q-Y, Ye S-B, et al. RNA sequencing of $\mathrm{Xp} 11$ translocation-associated cancers reveals novel gene fusions and distinctive clinicopathologic correlations. Mod Pathol Off J U S Can Acad Pathol Inc. 2018;31(9):1346-60. doi:10.1038/s41379-018-0051-5

[16] Inamura K. Translocation Renal Cell Carcinoma: An Update on Clinicopathological and Molecular Features. Cancers. 2017;9(9). doi:10.3390/cancers9090111

[17] Andeen N, Tretiakova M. MiT family translocation renal cell carcinomas. MiT family translocation renal cell carcinomas. Published March 28, 2020. Accessed July 15, 2020. http://www.pathologyoutlines.com/topic/kidneytumormali gnantASPLTFE3.html

[18] Raben N, Puertollano R. TFEB AND TFE3, LINKING LYSOSOMES TO CELLULAR ADAPTATION TO STRESS. Annu Rev Cell Dev Biol. 2016;32:255-78. doi:10.1146/annurev-cellbio-111315-125407

[19] Argani P. Summary \& Introduction - Translocation Renal Cell Carcinoma | Johns Hopkins Pathology. Translocation Renal Cell Carcinoma. Accessed July 13, 2020. https://path ology.jhu.edu/renal-cell-carcinoma/summary-introduction
[20] Argani P. MiT family translocation renal cell carcinoma. Semin Diagn Pathol. 2015;32(2):103-13. doi:10.1053/j. semdp.2015.02.003

[21] Sukov WR, Hodge JC, Lohse CM, et al. TFE3 rearrangements in adult renal cell carcinoma: clinical and pathologic features with outcome in a large series of consecutively treated patients. Am J Surg Pathol. 2012;36(5):663-70. doi:10.1097/PAS.0b013e31824dd972

[22] Komai Y, Fujiwara M, Fujii Y, et al. Adult Xp11 translocation renal cell carcinoma diagnosed by cytogenetics and immunohistochemistry. Clin Cancer Res Off $\mathbf{J}$ Am Assoc Cancer Res. 2009;15(4):1170-6. doi:10.1158/10780432.CCR-08-1183

[23] Rao Q, Chen J, Wang J, et al. Renal cell carcinoma in children and young adults: clinicopathological, immunohistochemical, and VHL gene analysis of 46 cases with follow-up. Int J Surg Pathol. 2011;19(2):170-9. doi:10.1177/1066896909354337

[24] Argani P, Olgac S, Tickoo SK, et al. Xp11 translocation renal cell carcinoma in adults: expanded clinical, pathologic, and genetic spectrum. Am J Surg Pathol. 2007;31(8):1149-60. doi:10.1097/PAS.0b013e318031ffff

[25] Wagner AJ, Goldberg JM, Dubois SG, et al. Tivantinib (ARQ 197), a selective inhibitor of MET, in patients with microphthalmia transcription factor-associated tumors: results of a multicenter phase 2 trial. Cancer. 2012;118(23):5894-902. doi:10.1002/cncr.27582

[26] Malouf GG, Camparo P, Oudard S, et al. Targeted agents in metastatic Xp11 translocation/TFE3 gene fusion renal cell carcinoma (RCC): a report from the Juvenile RCC Network. Ann Oncol Off J Eur Soc Med Oncol. 2010;21(9):1834-8. doi:10.1093/annonc/mdq029

[27] Choueiri TK, Lim ZD, Hirsch MS, et al. Vascular endothelial growth factor (VEGF)-targeted therapy for the treatment of adult metastatic Xp11.2 translocation renal cell carcinoma. Cancer. 2010;116(22):5219-25. doi:10.1002/cncr.25512

[28] Al-Daghmin A, Aboumohamed A, Din R, et al. Readmission After Robot-assisted Radical Cystectomy: Outcomes and Predictors at 90-Day Follow-up. Urology. 2014;83(2):350-6. doi:10.1016/j.urology.2013.09.056

[29] Boilève A, Carlo MI, Barthélémy P, et al. Immune checkpoint inhibitors in MITF family translocation renal cell carcinomas and genetic correlates of exceptional responders. J Immunother Cancer. 2018;6(1):159. doi: 10.1186/s40425-018-0482-z 